\title{
Evidence of Tandem Repeat and Extra Thiol-groups Resulted in the Polymeric Formation of Bovine Haptoglobin: A Unique Structure of Hp 2-2 Phenotype
}

\author{
Yi An Lai ${ }^{1}$, I Hsiang Lai ${ }^{1}$, Chi Feng Tseng ${ }^{1}$, James Lee ${ }^{1}$ and Simon J. T. Mao ${ }^{1,2, *}$ \\ ${ }^{1}$ Research Institute of Biochemical Engineering, Department of Biological Science and Technology, National Chiao Tung University, \\ Hsinchu, Taiwan, ROC \\ ${ }^{2}$ Department of Biotechnology and Bioinformatics, Asia University, Taichung, Taiwan, ROC
}

Received 9 July 2007, 27 August 2007

\begin{abstract}
Human plasma $\mathrm{Hp}$ is classified as 1-1, 2-1, and 2-2. They are inherited from two alleles $H p 1$ and $H p$, but there is only $H p 1$ in almost all the animal species. Hp 2-2 molecule is extremely large and heterogeneous associated with the development of inflammatory-related diseases. In this study, we expressed entire bovine $\mathrm{Hp}$ in $E$. coli as a $\alpha \beta$ linear form. Interestingly, the antibodies prepared against this form could recognize the subunit of native Hp. In stead of a complicated column method, the antibody was able to isolate bovine $\mathrm{Hp}$ via immunoaffinity and gelfiltration columns. The isolated $\mathrm{Hp}$ is polymeric containing two major molecular forms (660 and $730 \mathrm{kDa})$. Their size and hemoglobin binding complex are significantly larger than that of human Hp 2-2. The amino-acid sequence deducted from the nucleotide sequence is similar to human $H p 2$ containing a tandem repeat over the $\alpha$ chain. Thus, the $H p 2$ allele is not unique in human. We also found that there is one additional -SH group (Cys-97) in bovine $\alpha$ chain with a total of 8 -SH groups, which may be responsible for the overall polymeric structure that is markedly different from human $\mathrm{Hp} \mathrm{2-2.} \mathrm{The} \mathrm{significance}$ of the finding and its relationship to structural evolution are also discussed.
\end{abstract}

Keywords: Amino-acid sequence, Bovine and human haptoglobin, DNA evolution, Phenotype, Purification

\section{Introduction}

Haptoglobin $(\mathrm{Hp})$ is an acute phase protein, which appears in the plasma of all mammals (Wang et al., 2001; Faijmakers et

*To whom correspondence should be addressed. Te: 886-3-571-2121 ext. 56948; Fax: 886-3-572-9288

E-mail: mao1010@ms7.hinet.net; mhanb20012001@yahoo.com.tw al., 2003; Gervois et al., 2004) and the level rises in response to infection and inflammation. The most-noted biological functions of $\mathrm{Hp}$ are to capture released hemoglobin during excessive hemolysis (Kristiansen et al., 2001) and to scavenge the free radicals during oxidative stress (Tseng et al., 2004b).

Similar to blood types, Hp of human is classified as three phenotypes: $\mathrm{Hp} \mathrm{1-1,} \mathrm{Hp} \mathrm{2-1} \mathrm{and} \mathrm{Hp} \mathrm{2-2} \mathrm{(Schultze} \mathrm{and}$ Heremans, 1966). They are genetically inherited from Hp 1 and Hp 2 alleles corresponding to the formation of two polypeptide chains, $\alpha 1 \beta$ and $\alpha 2 \beta$, respectively. However, it has been thought that only one allele exists in other mammals (Hp 1-1). Following posttranslational cleavage on $\alpha \beta$ polypeptide, $\alpha$ and $\beta$ chains are formed and then linked by disulfide bridges producing the mature Hp (Fig. 1). Aminoacid sequence of the Hp 2 produced $\alpha 2$ chain (a large peptide containing 142 amino acids) is the same as the Hp 1 produced $\alpha 1$ (a small peptide containing 83 amino acids), but the $\alpha 2$ contains an additional repeated region (59 residues). This repeated region provides an extra - $\mathrm{SH}$ group which gives rise to the polymeric form 2-1 and 2-2 as depicted in Fig. 2 (Wejman et al., 1984). It has been reported that the polymeric forms are associated with the risk of diabetes, kidney failure, autoimmune, and cardiovascular diseases (Miyoshi et al., 1991; Lange, 1992; Levy et al., 2000; Hochberg et al., 2002).

The gene frequencies of human Hp present geographical differences. In Asia (Taiwan, China, Korea, and Japan), Hp 1 allele frequency of homozygotes $(\sim 8 \%)$ is lower than that in Africa, Europe, and North America ( $20 \%$ or greater). It has been proposed that the human Hp 2 originated from Hp 1 about 2 million years ago in India and then gradually displaced Hp 1 (Maeda, 1985; McEvoy and Maeda, 1988; Langlois and Delanghe, 1996). Presumably, $\alpha 2$ is a consequence of a nonhomologous crossing-over within the structural alleles (Hp 1) during meiosis (Maeda, 1985; McEvoy and Maeda, 1988). Thus, only humans possess additional Hp 2-1 and 2-2 phenotypes.

Bovine Hp has been identified as a large molecule with 
polymeric forms (Morimatsu et al., 1991; Morimatsu et al., 1992), but the molecular mechanism involved in such arrangement remains unknown. In this study, we cloned and expressed recombinant bovine $\mathrm{Hp}$ in the $E$. coli expression system. Recombinant $\mathrm{Hp}$ was made as an uncleaved linear $\alpha \beta$ chain and used for raising the mouse and rabbit polyclonal antibodies. These antibodies were able to recognize the native $\alpha$ or $\beta$ unit of bovine Hp or both. The IgG of rabbit antirecombinant Hp was then utilized for the preparation of an immunoaffinity column. The purified Hp shows a copy of $\alpha$ and $\beta$ chain on reduced SDS-PAGE, in which the molecular weight of $\alpha$ chain mimics that of human $\alpha 2$. Amino-acid sequence alignment (deducted from the nucleotide sequence) of bovine Hp reveals that the $\alpha$ chain possesses a unique tandem repeat. We suggest that Hp 2 allele is not unique in human, but the bovine tandem repeat is markedly different from that of human. Besides the sequence heterogeneity within the tandem repeat, bovine $\alpha$ chain is consisted of an additional -SH group which is responsible for even larger Hp polymers as compared with human. The significance and detailed analysis of the nucleotide sequence of bovine Hp and its relationship to evolution are also discussed.

\section{Materials and Methods}

Materials. Escherichia coli JM109, M15 [pREP4], the pQE30 expression vector, and the RNeasy Mini kit were purchased from Qiagen. Plasmid preparation and gel-extraction kits were purchased from BD Biosciences. The proofreading DNA-polymerase and dNTP were purchased from Invitrogen. All restriction enzymes were purchased from New England Biolabs. T4-DNA-ligase and isopropyl $\beta$-D-thiogalactopyranoside (IPTG) were purchased from Fermentas. Rabbit anti-Goat IgG and Goat anti-mouse $\operatorname{IgG}$ were purchased from Chemicon. The HiTrap chelating column, $\mathrm{CNBr}$ activated Sepharose 4B and nitrocellulose membrane were purchased from Amersham Biosciences. The P-2 polyacrylamide gel was purchased from Bio-Rad. The ProSieve color proteins of low molecular weight marker (10-120 kDa) were purchased from Cambrex Bio Science Rockland, while the high molecular weight marker $(97-584 \mathrm{kDa})$ were purchased from Sigma. All other chemicals were purchased from Sigma and Merck without any further purification. The buffers used in this report were all filtered through a $0.45 \mu \mathrm{m}$ filter before using. Bovine plasma of normal healthy Holstein cows (Bos taurus) were obtained from the County Livestock Disease Control Center of Pingtung, Taiwan.

Gene cloning and Plasmid construction. Total RNA was isolated and purified from the bovine (Bos taurus) liver using the RNeasy Mini kit with the procedures recommended by the manufacturer. An oligo(dT) 18 mer was used for the reverse transcription step. The first strand cDNAs were synthesized with M-MLV reverse transcriptase at $37^{\circ} \mathrm{C}$ for $50 \mathrm{~min}$. Gene fragments coding for bovine $\mathrm{Hp}$ was amplified by PCR using proofreading DNA-polymerase and oligonucleotide primers (Lai et al., 2007; Emamzadeh et al., 2006). The oligonucleotide primers prepared were 5'-TTCCTGCA GTGGAAACCGGCAGTGAGGCCA-3' (forward) and 5'-CGGAA
AACCATCGCTAACAACTAAGCTTGGG-3' (reverse). Both PstI and HindIII restriction sites were incorporated into the 5 ' end of the forward and reverse sequence primers. The cDNA of Hp was ligated into the PstI/HindIII sites of an E. coli expression vector, pQE30, similar to that described previously (Wang et al., 2002; Lai et al., 2007). The plasmids were screened in JM109 and then expressed in M15 (pREP4).

Expression of Bovine recombinant $\mathrm{Hp}$ and isolation of inclusion bodies. Escherichia coli [M15 (pREP4)] was transformed with the recombinant plasmid and cultured in $1.2 \mathrm{~L}$ of Luria-Bertani (LB) medium containing ampicillin $(100 \mu \mathrm{g} / \mathrm{mL})$ and kanamycin $(50 \mu \mathrm{g} /$ $\mathrm{mL}$ ) at $37^{\circ} \mathrm{C}$ on a rotary shaker (Wang et al., 2002; Lai et al., 2007). When the optical density reached 0.6 at wavelength $600 \mathrm{~nm}$, the expression of bovine recombinant $\mathrm{Hp}$ was then induced by $1 \mathrm{mM}$ IPTG at $37^{\circ} \mathrm{C}$ for $6 \mathrm{~h}$. The medium was centrifuged at $8,000 \mathrm{~g}$ for 5 min and washed three times in a wash buffer containing $20 \mathrm{mM}$ Tris- $\mathrm{HCl}, \mathrm{pH}$ 8.0. The induced cells were then suspended in $40 \mathrm{~mL}$ of wash buffer and sonicated for $5 \mathrm{~min}$ at $4^{\circ} \mathrm{C}$, followed by centrifugation at $20,000 \mathrm{~g}$ for $20 \mathrm{~min}$ at $4^{\circ} \mathrm{C}$. The pellet containing the inclusion body was resuspended in $40 \mathrm{~mL}$ of $2 \mathrm{M}$ urea containing $20 \mathrm{mM}$ Tris-HCl, $0.5 \mathrm{M} \mathrm{NaCl}, 2 \%$ Triton X-100, pH 8.0 and sonicated as above, followed by centrifugation at $20,000 \mathrm{~g}$ for $20 \mathrm{~min}$ at $4^{\circ} \mathrm{C}$. Finally, the pellet was washed twice in wash buffer and stored at $-20^{\circ} \mathrm{C}$.

Solubilization of expressed $\mathrm{Hp}$ from inclusion bodies. The inclusion bodies were dissolved in a binding buffer containing 20 $\mathrm{mM}$ Tris- $\mathrm{HCl}, 0.5 \mathrm{M} \mathrm{NaCl}, 5 \mathrm{mM}$ imidazole, $6 \mathrm{M}$ guanidine- $\mathrm{HCl}$, and $1 \mathrm{mM}$ 2-mercaptoethanol, $\mathrm{pH}$ 8.0. The mixture was gently stirred at $4^{\circ} \mathrm{C}$ for $12 \mathrm{~h}$ and the insoluble material was removed by centrifugation at $20,000 \mathrm{~g}$ for $20 \mathrm{~min}$ at $4^{\circ} \mathrm{C}$. Finally, the remaining soluble supernatant was passed through a syringe filter $(0.45 \mu \mathrm{m})$ and proceeded directly for purification and refolding.

Purification and refolding of expressed Hp. Purification of bovine expressed $\mathrm{Hp}$ was conducted using a nickel affinity column (Lai et al., 2007). Briefly, a 3 mL-HiTrap chelating column was washed with $15 \mathrm{~mL}$ distilled water. After loading $3 \mathrm{~mL}$ of $0.1 \mathrm{M}$ $\mathrm{NiSO}_{4}$, the column was extensively equilibrated with a binding buffer described above. The recombinant fusion protein was subsequently loaded onto the column and washed with the same buffer. The bound protein was then treated with a gradient, starting with $6 \mathrm{M}$ urea and finishing at a buffer without urea. Finally, the Hp were eluted using a $30 \mathrm{~mL}$ linear gradient starting with an elution buffer containing $20 \mathrm{mM}$ Tris- $\mathrm{HCl}, 0.5 \mathrm{mM} \mathrm{NaCl}, 20 \mathrm{mM}$ imidazole, and $1 \mathrm{mM}$ 2-mercaptoethanol, pH 8.0 and ending with the same buffer containing $300 \mathrm{mM}$ imidazole. Protein fractions were pooled and then desalted on a P-2 column equilibrated with $0.05 \mathrm{M}$ ammonium bicarbonate, followed by lyophilization. Protein concentration was determined using a Bio-Rad kit based on the Lowry method (Lowry et al., 1951), while using bovine serum albumin as a standard.

SDS-PAGE and Western blot analysis. Sodium dodecyl sulfatepolyacrylamide gel electrophoresis (SDS-PAGE) was performed according to Laemmli's method (Laemmli, 1970) with some modifications using $5 \%$ polyacrylamide $(\mathrm{w} / \mathrm{v})$ on the stacking gel 
as previously described (Yang and Mao, 1999). In general, the tested sample was preheated at $100^{\circ} \mathrm{C}$ for $10 \mathrm{~min}$ in a buffer containing $12 \mathrm{mM}$ Tris- $\mathrm{HCl}, 0.4 \%$ SDS, $5 \%$ glycerol, $2.9 \mathrm{mM} 2-$ mercaptoethanol, and $0.02 \%$ bromphenol blue, $\mathrm{pH} 6.8$ before loading to the gel. The samples were run for about $1.5 \mathrm{~h}$ at $100 \mathrm{~V}$ and stained using Coomassie brilliant blue R-250. Western blot analysis was performed similar to that described previously (Song et al., 2005; Xu et al., 2006b). In brief, the gel was electrotransferred to a nitrocellulose membrane at $90 \mathrm{~mA}$ for $1 \mathrm{~h}$ in a semi-dry transfer cell (Bio-Rad) containing a transfer buffer. The transferred membrane was then immersed in $2 \%$ gelatin (w/v) in PBS for $1 \mathrm{~h}$ at room temperature while shaking gently. After washing with PBS three times, $5 \mathrm{~min}$ each, the membrane was incubated with a primary rabbit or mouse polyclonal antibody against bovine Hp (1: 5000 dilution in PBS washing buffer containing $0.2 \%(\mathrm{w} / \mathrm{v})$ gelatin and $0.05 \%(\mathrm{v} / \mathrm{v})$ Tween 20 for $1 \mathrm{~h})$ at room temperature and washed three times for $5 \mathrm{~min}$ each. The membrane was then incubated with $1: 5,000$ diluted peroxidase-conjugated anti-IgG in washing buffer for $1 \mathrm{~h}$. In addition, the membrane was washed twice with washing buffer and further washed once with PBS. Finally, the membrane was developed using 3,3-diaminobenzidine (DAB) as a substrate for horseradish peroxidase.

\section{Preparation of polyclonal antibodies against bovine recombinant} Hp. Female Balb/c mice (5 to 7 weeks of age) and rabbits (8-10 months of age) were used for raising the antibody against recombinant Hp. In brief, each animal was immunized by subcutaneous and intraperitoneal (only for mouse) injections of purified bovine recombinant $\mathrm{Hp}$. About 1-2 $\mathrm{mg}$ of protein in sterile PBS were mixed and homogenized with an equal volume of Freund's complete adjuvant using a 3-way stopcock. A total emulsion of 500 $\mu \mathrm{L}$ containing $200 \mu \mathrm{g}$ antigen was injected subcutaneously onto the six sites of the animal back with or without one additional intraperitoneal injection for mice or rabbits, respectively. After 21 $\mathrm{d}$, each animal was boosted by 2 intramuscular injections with a total of $500 \mu \mathrm{L}$ (50\% sterile PBS solution and $50 \%$ incomplete adjuvant) containing $200 \mu \mathrm{g}$ of bovine recombinant $\mathrm{Hp}$. Seven to ten days following the final booster, blood was collected in $0.1 \%$ EDTA and plasma was obtained. All the procedures were conducted according to the regulation of National Science Council (NSC) and were approved by the Animal Study Group of the University. Titers and binding specificity were tested by ELISA and Western blotting, respectively. In general, the titers achieved were greater than $1: 10,000$ dilutions by an enzyme labeled immunosorbent assay (ELISA) (Chen et al., 2004; Xu et al., 2006). Additional booster injections were given when necessary.

Preparation of antibody affinity column. Dialyzed IgG fraction of rabbit polyclonal antibody prepared against recombinant $\mathrm{Hp}$ in a coupling buffer $\left(0.1 \mathrm{M} \mathrm{NaHCO}_{3}\right.$ and $\left.0.5 \mathrm{M} \mathrm{NaCl}, \mathrm{pH} 8.3\right)$ was first coupled to $\mathrm{CNBr}$-activated Sepharose-4B according to the method recommended by manufacturer as previously described (Tseng et al., 2004a; Yueh et al., 2007). Briefly, $5 \mathrm{~g}$ of freeze-dried Sepharose ( $1 \mathrm{~g}$ of freeze-dried powder gave about $3.5 \mathrm{~mL}$ final volume of gel) was swollen and suspended in $1 \mathrm{mM} \mathrm{HCl}$ and immediately washed with $20 \times$ volume of the same solution within $15 \mathrm{~min}$ on a sintered glass filter. The gel was then washed with a coupling buffer and degassed. About $15 \mathrm{~mL}(6 \mathrm{mg} / \mathrm{mL})$ of dialyzed rabbit anti-recombinant
Hp IgG in coupling buffer was slowly added into the gel (CNBractivated Sepharose-4B), while gently rotating for $1 \mathrm{~h}$ at room temperature. After which time, the gel was washed with $5 \times$ volume of coupling buffer to remove unbound materials via a sintered glass filter. The gel was then treated with a blocking solution containing $0.1 \mathrm{M}$ Tris- $\mathrm{HCl}$ buffer, $\mathrm{pH} 8.0$, for $2 \mathrm{~h}$ at room temperature to saturate the remaining reactive-sites. The degassed gel was then washed with 3 cycles of $0.1 \mathrm{M}$ acetate buffer containing $0.5 \mathrm{M}$ $\mathrm{NaCl}, \mathrm{pH} 4$ and $0.1 \mathrm{M}$ Tris- $\mathrm{HCl}$ containing $0.5 \mathrm{M} \mathrm{NaCl}, \mathrm{pH} 8$. Finally, the gel was equilibrated in PBS and packed onto a $1.5 \times 25$ $\mathrm{cm}$ column with a bed volume of approximately $17.5 \mathrm{~mL}$ of coupled Sephrose.

Purification of bovine native Hp using antibody affinity-column chromatography. Hp positive bovine plasma was first confirmed by a Western blot. Typically, $1.5 \mathrm{~mL}$ of filtered bovine plasma was loaded onto the antibody affinity-column and allowed to enter into the top of the Sepharose at room temperature. After $1 \mathrm{~h}$ incubation, the unbound plasma materials were eluted and washed with $45 \mathrm{~mL}$ PBS, pH 7.4, and then eluted with $45 \mathrm{~mL}$ of a freshly prepared PBS with $\mathrm{pH} 11$ adjusted by ammonia. Three milliliters of each fraction was collected in a tube containing $0.1 \mathrm{~mL}$ of $1 \mathrm{M}$ Tris- $\mathrm{HCl}$ buffer, $\mathrm{pH} 6.8$, to immediately neutralize the $\mathrm{pH}$ value. Pooled fractions containing $\mathrm{Hp}$ were then concentrated to a final volume of $0.2 \mathrm{~mL}$ using an Amicon centrifugal filter (Millipore).

Further purification of bovine native $\mathrm{Hp}$ by gel filtration column. Concentrated solution of affinity-isolated bovine Hp was filtered through a $0.45 \mu \mathrm{m}$ nylon fiber prior to high performance liquid chromatography (HPLC). The HPLC system (Waters) consisted of two pumps, an automatic sample injector, and a photodiode array detector. Superose-12 column $(1 \times 30 \mathrm{~cm})$ (Pharmacia) was used for further purification of affinity-isolated $\mathrm{Hp}$. The column was preequilibrated with $50 \mathrm{mM}$ ammonium bicarbonate. Affinity-isolated $\mathrm{Hp}(0.8 \mathrm{~mL})$ was chromatographed on the column at a flow rate of $0.4 \mathrm{~mL} / \mathrm{min}$. Fractions containing bovine native Hp, identified by an ELISA and a Western blot, were pooled and concentrated to a final volume of $1 \mathrm{~mL}$ using an Amicon centrifugal filter and then lyophilized. The lyophilized bovine native $\mathrm{Hp}$ was stored at $-80^{\circ} \mathrm{C}$ until analyzing.

Phenotyping. Hp phenotype was determined by a native-PAGE using hemoglobin-supplemented serum or plasma (Liau et al., 2003). Briefly, $6 \mu \mathrm{L}$ of plasma were first incubated with $3 \mu \mathrm{L}$ of 5 $\mathrm{mg} / \mathrm{mL}$ hemoglobin at room temperature for $10 \mathrm{~min}$ and then equilibrated with $3 \mu \mathrm{L}$ of sample buffer (containing $0.625 \mathrm{M}$ Tris$\mathrm{HCl}, \mathrm{pH} 6.8,50 \%$ (v/v) glycerol, and $0.125 \mathrm{mg} / \mathrm{L}$ bromphenol blue). The reaction mixture was run on a step-gradient polyacrylamide gel ( $\mathrm{pH} 8.8$ ). The bottom gel layer containing a 7\% polyacrylamide, while a $5 \%$ polyacrylamide (26.5: 1 acrylamide: bis-acrylamide) was employed as a top stacking gel ( $\mathrm{pH}$ 6.8). Electrophoresis was conducted at an initial voltage of $120 \mathrm{~V}$ and increased to $150 \mathrm{~V}$ when the dye front reached the separating gel. After the electrophoresis, the Hp-hemoglobin complexes were visualized by shaking the gel in freshly prepared peroxidase substrate $(0.5 \mathrm{~mL}$ of dimethyl sulfoxide, containing $25 \mathrm{mg}$ of 3,3'-Diaminobenzidine, $49 \mathrm{~mL}$ of phosphatebuffered saline, and $100 \mu \mathrm{L}$ of $30 \%$ hydrogen peroxide). 


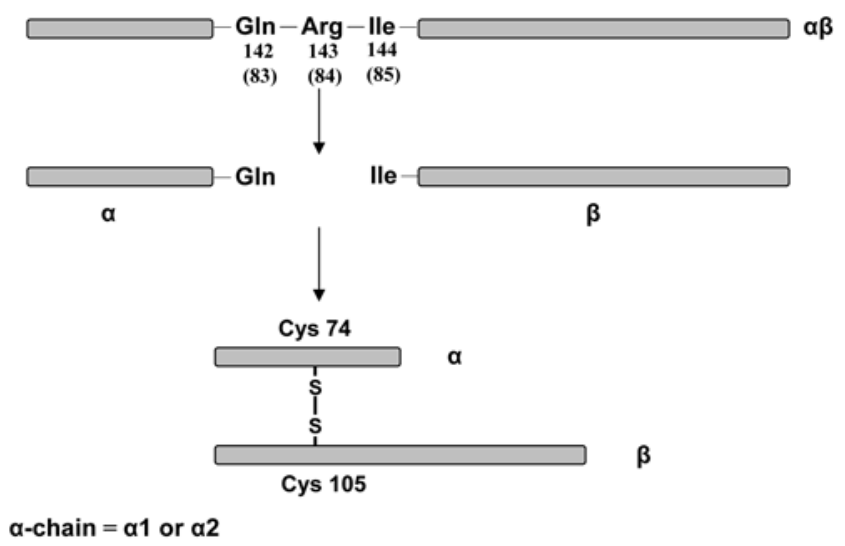

a1-chain

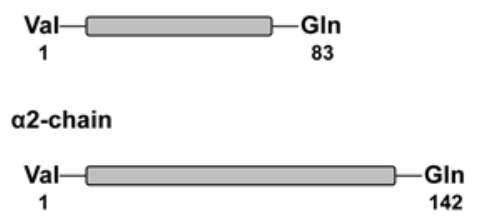

Fig. 1. Posttranslational cleavage between the Arg of $\alpha$ chain and Ile of $\beta$ chain of human Hp. Following the posttranslational cleavage, the Arg residue is removed forming one single $\alpha$ and $\beta$ chain. Eventually, the mature $\mathrm{Hp}$ is formed by the disulfide linkages between $\alpha$ and $\beta$ chain with a basic $\alpha-\beta$ subunit. Depending on the Hp allele, the $\alpha$ chain can be classified as $\alpha 1$ and $\alpha 2$ containing 83 and 142 amino-acid residues, respectively.

Others. Human Hp 1-1, 2-1, and 2-2 were obtained using the procedures previously described (Tseng et al., 2004a).

\section{Results}

Cloning and expression of recombined bovine Hp in $E$. coli. We constructed the plasmid using pQE30 as a vector and transformed it into $E$. coli. Protein extract of transformed cells induced by $1 \mathrm{mM}$ IPTG revealed that bovine $\mathrm{Hp}$ was efficiently and highly expressed in $E$. coli, in which recombinant $\mathrm{Hp}$ accounted for more than $40 \%$ of the total cellular proteins as analyzed by SDS-PAGE in a reduced condition (Fig. 3, Lanes 1 and 2). Because substantial amount of recombinant Hp was found in the inclusion bodies, they were solubilized in $8 \mathrm{M}$ urea prior to the isolation. Following renaturation using a ureagradient from 6 to $0 \mathrm{M}$ directly on a nickel affinity column, the $\mathrm{Hp}$ was then eluted using $300 \mathrm{mM}$ imidazole. The isolated $\mathrm{Hp}$ shows a single band on SDS-PAGE with an apparent molecular weight of approximately $50 \mathrm{kDa}$ (Fig. 3, Lane 3). This result displays that expressed bovine recombinant $\mathrm{Hp}$ is not cleaved into $\alpha$ and $\beta$ subunits indicating that $E$. coli contains no $\mathrm{C} 1 \mathrm{r}-\mathrm{like}$ protease (Wicher and Fries, 2004) that is responsible for the cleavage of $\alpha$ and $\beta$ chains in mammalian cells (Fig. 1).

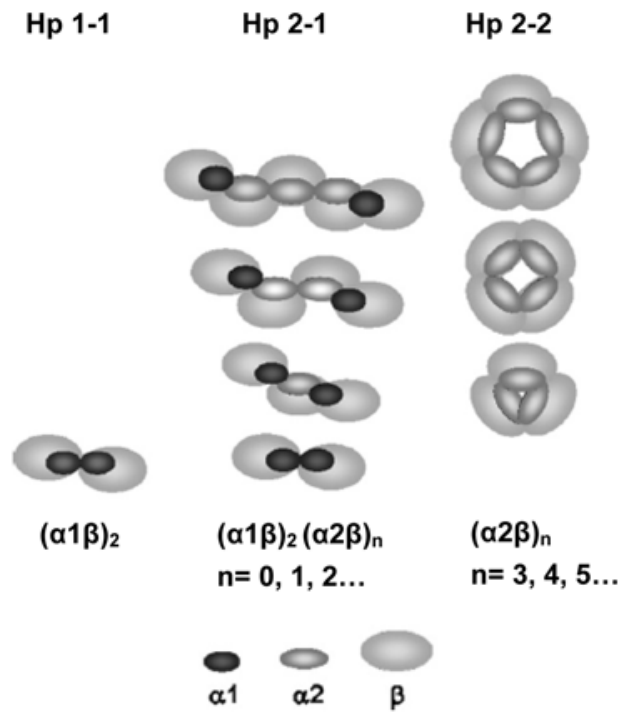

Fig. 2. Schematic drawing of molecular arrangement in human Hp phenotypes. Hp 1-1 possesses the simplest homodimer $(\alpha 1 \beta)_{2}$, whereas Hp 2-1 is comprised of polymeric structures in a linear form: a homodimer $(\alpha 1 \beta)_{2}$, a trimer $(\alpha \beta)_{3}$, and other polymers. Here, $\alpha$ represents both $\alpha 1$ and $\alpha 2$ chains. Hp 2-2 is comprised of cyclic structures: a trimer $(\alpha 2 \beta)_{3}$ and other cyclic polymers. Each $\alpha 1, \alpha 2$, or $\beta$ contains 83,142 or, or 243 amino-acid residues, respectively.

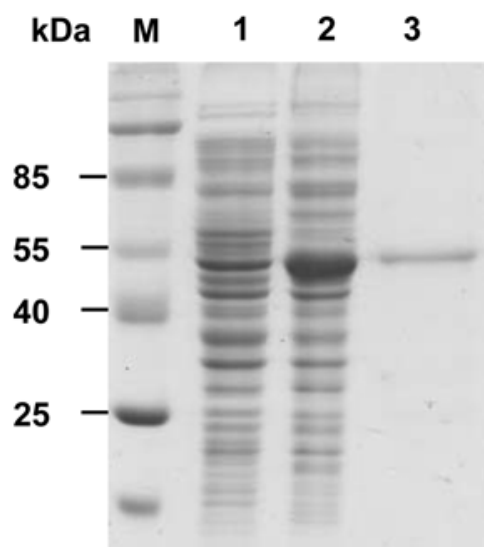

Fig. 3. Expression of recombinant bovine $\mathrm{Hp}$ in E. coli. Lane M, molecular markers; Lane 1, whole normal cell lysate; Lane 2, whole cell lysate containing expressed bovine $\mathrm{Hp}$; Lane 3, purified recombinant bovine Hp. Cells were incubated at $37^{\circ} \mathrm{C}$ for $6 \mathrm{~h}$ in the presence of the induction agent IPTG. SDS-PAGE gel (12\%) was loaded with samples containing $1 \%$ mercaptoethanol.

Characterization of mouse and rabbit polyclonal antibodies prepared against bovine recombinant Hp. We prepared polyclonal antibodies against recombinant $\mathrm{Hp}$ (a single $\alpha \beta$ polypeptide) in mice and rabbits to test whether the antibodies could recognize plasma native Hp (a cross-linked $\alpha-\beta$ subunit). Interestingly, we found mouse recombinant $\mathrm{Hp}$ antibody to be able to react with both native $\alpha$ and $\beta$ chains on a Western blot, whereas rabbit antibody only reacted with the $\alpha$ chain (Fig. 4). 




Fig. 4. Western blot of bovine plasma using mouse or rabbit polyclonal antibodies prepared against bovine recombinant Hp. Bovine plasma $(1 \mu \mathrm{L})$ containing $1 \%$ mercaptoethanol was loaded on a $10-15 \%$ SDS-PAGE gradient gel. Lane M, molecular markers; Lane 1, developed by mouse anti-bovine recombinant $\mathrm{Hp}$; Lane 2, developed by rabbit anti-bovine recombinant Hp. Notably, mouse antibody recognizes both $\alpha$ and $\beta$ chains, while rabbit antibody only recognizes the $\alpha$ chain of bovine $\mathrm{Hp}$.

Determination of the presence of $\mathrm{Hp}$ in bovine plasma. In contrast to humans, it has been reported that bovine Hp is usually not expressed in the plasma (Conner et al., 1988; Morimatsu et al., 1992; Yoshino et al., 1992; Petersen et al., 2004). We randomly collected plasma from 50 normal healthy cows and determined whether Hp was present in their plasma using a Western blot. Notably, approximate $40 \%$ of the cows evaluated possessed plasma Hp (Fig. 5). Among those who possessed Hp, there was only one single phenotype as judged by the molecular pattern of $\alpha$ chain (Fig. 5).

Purification of bovine native Hp. With respect to the isolation of bovine Hp, a method of using HPLC system combined with anion-exchange, Sephacryl S-300, TSK Phenyl5PW, and TSK DEAE-5PW columns together has been reported (Morimatsu et al., 1991). The procedures however are somewhat complicated and difficult to perform and timeconsuming. The yield of final isolated protein is also relatively low. To test whether we could take the advantage of the rabbit polyclonal antibody prepared against linear form of recombinant $\mathrm{Hp}$ (a single $\alpha \beta$ polypeptide), we attempted to isolate Hp from bovine plasma using an immunoaffinity column. Plasma of cows showing high levels of Hp (Fig. 5) were pooled and applied onto the column using a procedure recommended by the manufacturer. A typical affinity column chromatograph is shown in Fig. 6A, in which a major bound protein was eluted at $\mathrm{pH} 11$. This fraction contained approximately $60 \%$ of bovine $\mathrm{Hp}$, as analyzed on a SDS-PAGE in reducing condition. Following another gel-filtration using an HPLC column (Superpose-12) (Fig. 6B), the purification of bovine Hp was achieved with homogeneity almost greater than 90\% (Fig. 6C). To identify its phenotype, the isolated bovine Hp was
(A)

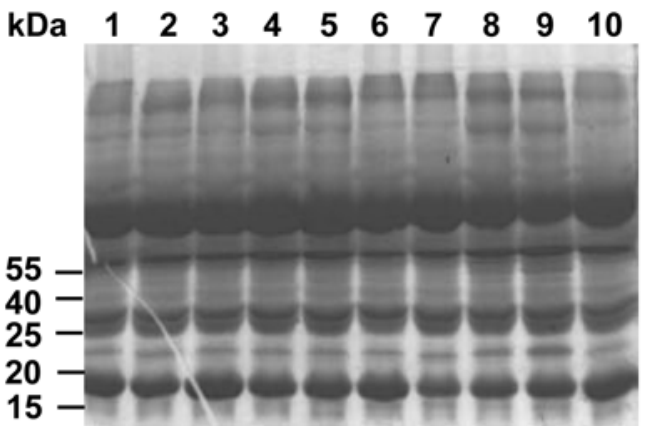

(B) 55

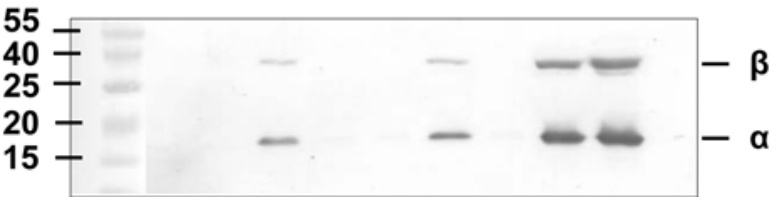

Fig. 5. Typical example of a Western blot of bovine plasma in reducing condition. Normal healthy cow plasma $(1 \mu \mathrm{L})$ was loaded on a $10-15 \%$ SDS-PAGE gradient gel. A: Coomassie blue staining; $\mathrm{B}$ : Western blot using mouse anti-recombinant $\mathrm{Hp}$. The data indicate approximately $40 \%$ of the normal cows $(n=50)$ expressed Hp in this study.

compared with human Hp 1-1, 2-1, and 2-2 standards. Clearly, bovine Hp was comprised of one $\beta$ and one $\alpha$ chain with a molecular weight similar to that of human $\alpha 2$ (Fig. 6C). We, therefore, tentatively classified bovine phenotype as Hp 2-2.

Comparison of bovine Hp with human Hp 2-2 using SDSPAGE. The putative bovine Hp 2-2 was further characterized on a $4 \%$ SDS-PAGE without a reducing reagent. Fig. 7A demonstrates that bovine $\mathrm{Hp}$ is consisted of at least two major molecular forms with molecular weight greater than $600 \mathrm{kDa}$. Using commercially available molecular markers and human Hp 2-2 as a calibrator (Fig. 7B), the estimated molecular weight of the two major forms of bovine $\mathrm{Hp}$ is about 660 and $730 \mathrm{kDa}$. These polymers totally differ from that of human $\mathrm{Hp}$ 2-2 which shows typical polymers following $(\alpha \beta)_{\mathrm{n}}$; where $\mathrm{n}=3,4,5$, --- up to 10 in this case. Of notice, $(\alpha \beta)_{3}$ was ran out of the gel (Fig. 7B). It remains ambiguous as to whether bovine Hp could be designated as a typical Hp 2-2.

Binding ability of isolated and native bovine $\mathrm{Hp}$ to hemoglobin. In the next experiment, we compared the hemoglobin binding ability of isolated bovine $\mathrm{Hp}$ with native Hp present in the plasma using a native-PAGE (5-7\% step gradient). Fig. 8 reveals that the formation of hemoglobin complex of isolated bovine $\mathrm{Hp}$ consisted of two large molecular forms that were almost identical to that of native $\mathrm{Hp}$ in bovine plasma. This result suggests that the Hp isolated under our experimental condition did not alter its molecular form and essentially retained the hemoglobin binding ability. The "size" of the bovine Hp-hemoglobin complex was markedly greater than that of human Hp 2-1 and 2-2 (Fig. 8). 
(A)

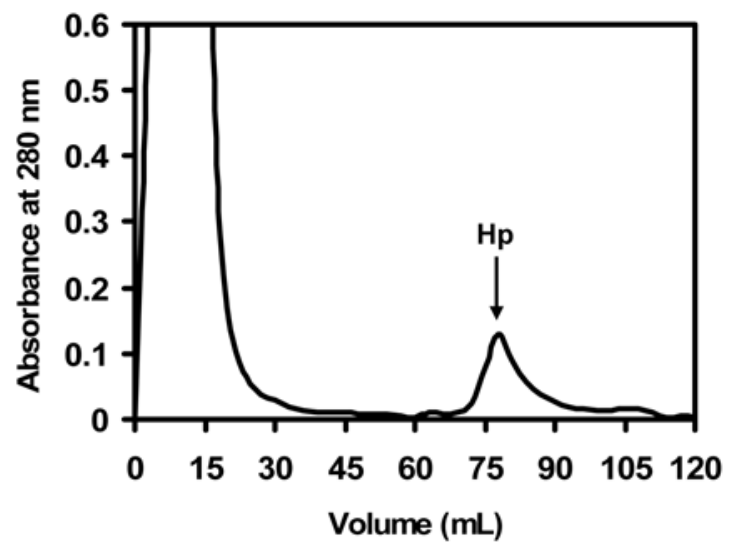

(B)

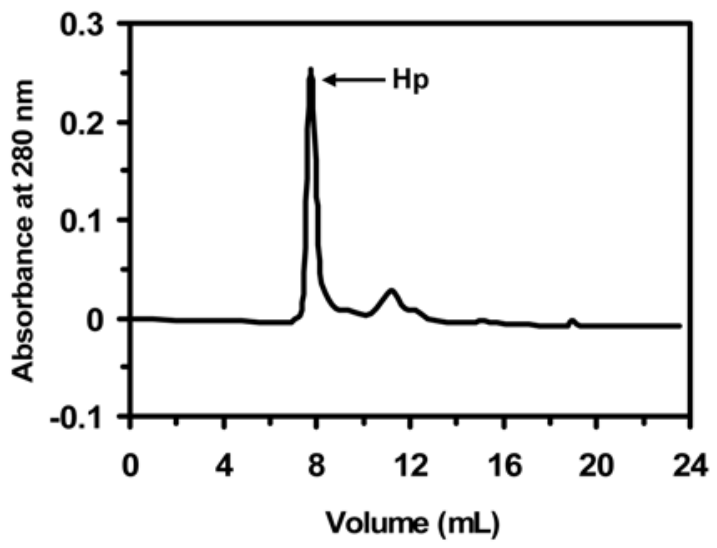

(C)

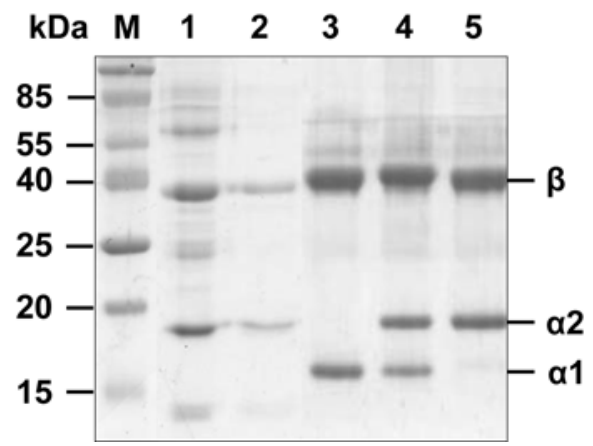

Fig. 6. Purification of bovine Hp using immunoaffinity column chromatography. A: For each chromatography, approximate $1.5 \mathrm{~mL}$ of bovine plasma (pooled from those with high expression of $\mathrm{Hp}$ ) were applied onto an immunoaffinity column and extensively washed with PBS, $\mathrm{pH}$ 7.4. The bound $\mathrm{Hp}$ was then eluted by a buffer at $\mathrm{pH}$ 11. B: Pooled fraction A was re-chromatographed on a gel-filtration HPLC (Superose-12; $1 \times 30 \mathrm{~cm}$ ) for further purification. C: Homogeneity of final purified Hp on SDS-PAGE. Lane M, molecular markers; Lane 1, isolated bovine Hp from affinity column; Lane 2, final isolated bovine Hp from an additional separation on HPLC column; Lanes 3-5, purified human Hp 1-1, 21 , and 2-2, respectively, represent as a reference for molecular weight evaluation.

Sequence alignment of bovine and human Hp 2-2. To further define that cow exhibits the "Hp 2-2" phenotype, the cloned bovine Hp cDNA was sequenced (Fig. 9). Amino-acid
(A)

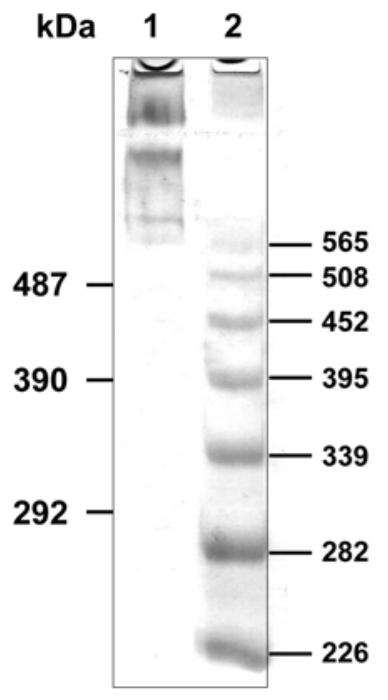

(B)

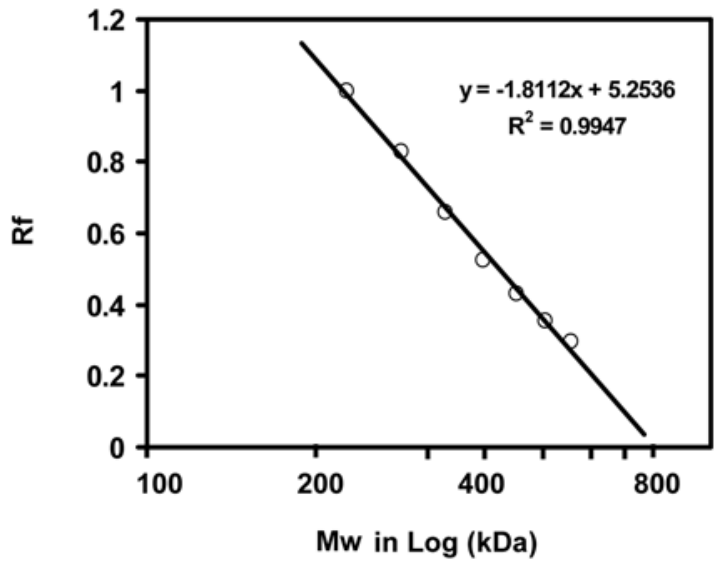

Fig. 7. Molecular weight characterization of purified bovine and human Hp 2-2 on 4\% non-reducing SDS-PAGE. Electrophoresis was conducted for $3.5 \mathrm{~h}$ at $20 \mathrm{~mA}$. A: Lane 1, isolated bovine $\mathrm{Hp}$; Lane 2, purified human Hp 2-2. Molecular weight marked on the left margin (in $\mathrm{kDa}$ ) represents those evaluated from a commercial source (gel not shown). Those marked on the right margin represent the true molecular weight calculated from the polymeric forms $(\alpha 2 \beta)_{n}(n=4-10)$ of human Hp 2-2, in which the Hp trimer $(n=3)$ was running out of the gel. B: Linear plot of the molecular weight of human $\mathrm{Hp}$ 2-2 polymers. Extrapolation from the plot, the molecular weight of two major forms of bovine Hp is approximately of 660 and $730 \mathrm{kDa}$.

sequence alignment, deducted from the nucleotide sequence, reveals that the putative sequence of bovine $\mathrm{Hp}$ is somewhat similar to human $\mathrm{Hp} \mathrm{2-2}$ with $80 \%$ and $68 \%$ sequence homology for $\beta$ and $\alpha$ chain, respectively (Fig. 9).

In humans, the $\alpha$ chain is responsible for the determination of Hp phenotypes. The Hp 1-1 or 2-2 homozygote is consisted of $\alpha 1$ ( 83 amino acids) or $\alpha 2$ (142 amino acids) chains, while Hp 2-1 is a heterozygote consisting of one $\alpha 1$ and one $\alpha 2$. As shown in Fig. 10 (by simplified $A B C$ domains), the uniqueness of human $\alpha 2$ is that it not only contains identical 


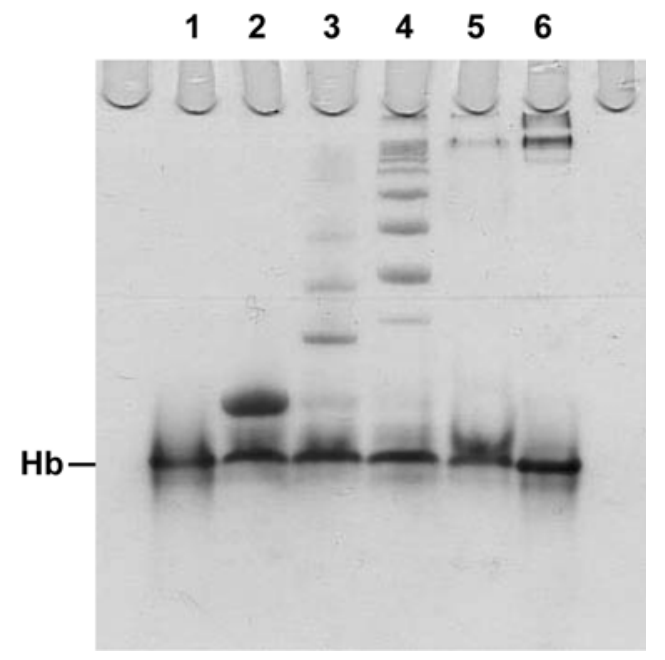

Fig. 8. Hemoglobin-binding property of isolated and native bovine plasma Hp. Bovine and human plasma were incubated with hemoglobin at room temperature for $30 \mathrm{~min}$ before conducting a $4 \%$ native-PAGE (see Materials and Methods). Lane 1, hemoglobin only; Lanes 2, 3, and 4, human plasma of Hp 1-1, 2-1, and 2-2 with hemoglobin, respectively; Lane 5, bovine plasma with hemoglobin; Lane 6, isolated bovine $\mathrm{Hp}$ with hemoglobin.

ABC domains of $\alpha 1$, but also an additional insertion of a redundant sequence (B1) between amino acid residues Asp-12 and Ala-70 (total 59 residues). The sequence homology between $\mathrm{B} 1$ and $\mathrm{B}$ in the repeat region is $96 \%$ with only two amino acids mutated. In cows, there also exists a repeat sequence within the $\alpha$ chain, but the sequence homology between the two repeat units (B1 and B) is 68\% (Fig. 10), which significantly differs from that of human with $98 \%$ identity. Accordingly, we designated bovine $\mathrm{Hp} \alpha$ genotype being unique as $\alpha 2$.

\section{Discussion}

Expression of bovine Hp. In the present study, we have shown bovine recombinant $\mathrm{Hp}$ expression in E. coli being abundant ( $>50 \%$ of the total endogenous proteins) and was mostly located in the inclusion bodies. The expressed bovine recombinant $\mathrm{Hp}$ being not cleaved into $\alpha$ and $\beta$ subunits in $E$. coli was expected, since $\mathrm{C} 1 \mathrm{r}$-like protease, responsible for the cleavage of $\alpha$ and $\beta$ chain in those mammalian cells (Wicher and Fries, 2004), is not present in E. coli (Lai et al., 2007). However, it makes the isolation of recombinant Hp relatively simple when using a nickel-affinity column in the presence of urea (Fig. 3). One advantage in expressing the $\mathrm{Hp}$ in $E$. coli is that the resulting protein (in an intact $\alpha \beta$ polypeptide chain) can be utilized for the preparation of polyclonal antibodies that in turn can be used as a probe for Western blot (Fig. 5) and for bovine Hp purification (Fig. 6).
Isolation of bovine native Hp. Rather than using combined anion-exchange, Sephacryl S-300, TSK Phenyl-5PW, and TSK DEAE-5PW columns for the isolation of bovine plasma Hp (Morimatsu et al., 1991); we used a straightforward purification method achieved by an affinity column coupled with the polyclonal antibody prepared against bovine recombinant Hp, followed by HPLC gel-filtration (Fig. 6). The latter procedures are essentially simple; it can be routinely utilized for the purification of bovine Hp. Notably, the estimated molecular weight of the two major forms of bovine $\mathrm{Hp}$ we isolated is about 660 and $730 \mathrm{kDa}$ (Fig. 7), which differs from previously reported values (1000-2000 $\mathrm{kDa}$ ). The reason for this discrepancy is readily not known, but one of the explanations is that there were at least four chromatographic procedures involved in the former purification method (Morimatsu et al., 1991). Bovine Hp might be reoxidized via the disulfide linkages (described below). It is of interest that the two large molecular forms we isolated could form hemoglobin complexes with the electrophoretic property almost identical to that of the native Hp that exists in the bovine plasma (Fig. 8). This result suggests that the Hp isolated under our experimental condition did not produce an observable alteration.

Presence of Hp in cow plasma. Hp level is abundant in human plasma with an approximate concentration of $100 \mathrm{mg} /$ dL (Dobryszycka, 1997). It has been reported that $\mathrm{Hp}$ is not present in normal healthy cow plasma (Conner et al., 1988; Morimatsu et al., 1992; Yoshino et al., 1992; Petersen et al., 2004), but was inducible by giving inflammatory reagents (Petersen et al., 2004). However, a recent report using immunoassay (Western blot and ELISA) indicated that Hp is present in cattle with a mean concentration of $8.4 \pm 3.4 \mathrm{mg} / \mathrm{dL}$ (Chan et al., 2004). The absence of plasma $\mathrm{Hp}$ in the latter investigation, however, is not particularly mentioned. In the present study, we show that about $40 \%$ of normal cows possess $\mathrm{Hp}$ (assessed by Western blot) in their plasma indicating that the presence of $\mathrm{Hp}$ in bovine plasma is dependent on the individuals (Fig. 5). Nevertheless, plasma of cows should be prescreened for Hp levels in order to isolate Hp. This can be simply done using a hemoglobin binding assay we described as the entire assay can be virtually finished within $60 \mathrm{~min}$ (Fig. 8). Undoubtedly, the factor(s) influencing the expression of plasma $\mathrm{Hp}$ in the normal cows remains unclear and deserves future investigations.

Primary structure of bovine $\alpha$ chain and its relationship to Hp polymers. It is worth mentioning that during the investigation of this work, a cDNA sequence of bovine $\mathrm{Hp}$ had also been filed in the NCBI database (GenBank accession no. NM_001040470), although a follow-up of structural analysis and its significance were not conducted.

As judged by our cDNA sequence of bovine $\mathrm{Hp} \alpha$ chain in the present study, there are several lines of evidence to support 


\section{(A)}

\section{Nucleotide sequence of bovine Hp $\beta$-chain}

\begin{tabular}{lr}
\hline ATCATCGGTGGCTCATTGGATGCCAAGGGCAGCTTTCCCTGGCAGGCCAAGATGGTCTCCCAGCATAACC & 70 \\
TCATCTCGGGAGCCACGCTCATCAATGAACGATGGCTCCTCACCACAGCTAAAAATCTCTACCTGGGTCA & 140 \\
CAGTAGTGACAAAAAAGCAAAGAACATCACTCCTACTTTAAGACTCTATGTGGGGAAGAACCAGCTTGTA & 210 \\
GAGGTGGAGAAGGTGGTTCTCCACCCTGACCACTCCAAGGTAGACATTGGGCTCATCAAACTCAGACAGA & 280 \\
AGGTACCTGTCAATGACAAAGTAATGCCCATCTGCCTACCTTCAAAAGATTATGTGAAGGTGGGTCGTGT & 350 \\
GGGTTATGTG TCTGGCTGGGGGCGAATGAAAACTTCAACTTTACGGAGCATCTGAAGTATGTCATGCTA & 420 \\
CCTGTGGCTGACCAAGACAAGTGTGTGAAACACTATGAGGGCGTCGACGCACCTAAAAATAAGACAGCTA & 490 \\
AGAGCCCCGTAGGGGTGCAACCCATACTGAATGAGAACACCTTCTGCGTCGGCCTGTCCAAGTACCAGGA & 560 \\
CGACACCTGCTATGGCGACGCCGGCAGCGCCTTCGTCGTTCACGACAAGGAAGACGACACCTGGTATGCG & 630 \\
GCCGGGATCCTGAGCTTTGACAAGAGCTGTGCTGTGGCTGAGTATGGTGTGTACGTGAAGGTGACCTCCA & 700 \\
TTCTGGACTGGGTTCGGAAAACATCGCTAACAAC & 735 \\
\hline
\end{tabular}

Putative amino-acid sequence alignment between bovine and human Hp $\beta$-chain

\begin{tabular}{|c|c|c|}
\hline $\begin{array}{l}\text { Bovine } \mathrm{Hp} \\
\text { Human } \mathrm{Hp} \mathrm{2-2}\end{array}$ & $\begin{array}{l}\text { IIGGSLDAKGSFPWQAKMVSQHNLISGATLINERWLLTTAKNLYLGHSSDKKAKDITPTL } \\
\text { ILGGHLDAKGSFPWQAKMVSHHNLTTGATLINEQWLLTTAKNLFLNHSENATAKDIAPTL }\end{array}$ & $\begin{array}{l}60 \\
60\end{array}$ \\
\hline Bovine $\mathrm{Hp}$ & RLYVGKNQLVEVEKVVLHPDHSKVDIGLIKLRQKVPVNDKVMPICLPSKDYVKVGRVGYV & 120 \\
\hline Human $\mathrm{Hp} 2-2$ & TLYVGKKQLVEIEKVVLHPNYSQVDIGLIKLKQKVSVNERVMPICLPSKDYAEVGRVGYV & 120 \\
\hline Bovine $\mathrm{Hp}$ & SGWGRNENFNFTEHLKYVMLPVADQDKCVKHYEGVDAPKNKTAKSPVGVQPILNENTFCV & 180 \\
\hline Human $\mathrm{Hp}$ 2-2 & SGWGRNANFKFTDHLKYVMLPVADQDQCIRHYEGSTVPEKKTPKSPVGVQPILNEHTFCA & 180 \\
\hline Bovine $\mathrm{Hp}$ & GLSKYQDDTCYGDAGSAFVVHDKEDDTWYAAGILSFDKSCAVAEYGVYVKVTSILDWVRK & 240 \\
\hline Human $\mathrm{Hp} 2-2$ & GMSKYQEDTCYGDAGSAFAVHDLEEDTWYATGILSFDKSCAVAEYGVYVKVTSIQDWVQK & 240 \\
\hline & TIANN & 245 \\
\hline Human $\mathrm{Hp} 2-2$ & TIAEN & 245 \\
\hline
\end{tabular}

(B)

Nucleotide sequence of bovine Hp a-chain

\begin{tabular}{lr}
\hline GTGGAAACCGGCAGTGAGGCCACAGCCGACAGCTGCCCAAAGGCCCCCGAGATTGCTAATAGCCATGTGG & 70 \\
AGTACTCGGTTCGCTATCAGTGTGACAAATATTACAAACTGCATGCTGGAAATGGGGTGTATACTTTTAA & 140 \\
CAATAAGCAATGGATAAACAAGGACATTGGACAGCAACTTCCTGAATGTGAAGAAGATGACAGCTGCCCA & 210 \\
GAGCCCCCCAAGATTGAAAATGGCTACGTGGAGTACTTGGTTCGCTATCAGTGCAAACCCTATTACACAC & 280 \\
TGCGCACCTGTGGAGATGGAGTGTACACCTTTAACAGTAAGAAGCAGTGGATAAATAAGAACATTGGACA & 350 \\
GAAACTCCCTGAATGTGAGGCAGTGTGCGGGAAGCCCAAGCACCCCGTGGACCAGGTGCAG & 411
\end{tabular}

Putative amino-acid sequence alignment between bovine and human $\mathrm{Hp} \alpha$-chain

\begin{tabular}{|c|c|c|}
\hline Bovine $\mathrm{Hp}$ & $\begin{array}{l}\text { B1 } \\
\text { VETGSEAT- - ADSCPKAPEIANSHVEYSVRYQCDKYYKLHA -GNGVYTFNNK -QWINKD }\end{array}$ & 55 \\
\hline Human $\mathrm{Hp} 2-2$ & VDSGNDVTDIADDGCPKPPEIAHGYVEHSVRYQCKNYYKLRTEGDGVYTLNDKKQWINKA & 60 \\
\hline Bovine $\mathrm{Hp}$ & $\begin{array}{l}\text { B } \\
\text { IGQQLPECEEE DDSCPEPPKIENGYVEYLVRYQCKRYYTLRTCGGDGVYTFNSKKQWINKNI }\end{array}$ & 115 \\
\hline Human $\mathrm{Hp} 2-2$ & VGDKLPEC EADDGCPKPPEIAHGYVEHSVRYQCKNYYKLRTEGDGVYTLNNEKQWINKAV & 120 \\
\hline Bovine $\mathrm{Hp}$ & GQKLPECEAMCGKKPKHPVDQVQ & 137 \\
\hline Human $\mathrm{Hp} 2-2$ & GDKLPECEAVCGKPKNPANPVQ & 142 \\
\hline
\end{tabular}

Fig. 9. Nucleotide sequence of bovine $\mathrm{Hp}$ and its putative amino-acid sequence alignment between human and bovine $\beta$ (A) and $\alpha$ (B) chains. The alignment, deducted from the nucleotide sequence, was constructed using ClustalW (Labarga et al., 2005; Pillai et al., 2005). Repeated sequences within the $\alpha$ chain are shown in the brackets with two distinct regions. Locations of cysteine residues (in shaded brackets) are almost the same between the two species, except that bovine Hp $\alpha$ chain has an additional Cys-97 in a total of 8 -SH groups. This extra -SH group (Cys-97) might be responsible for the formation of a larger bovine Hp polymer than that of human Hp 2-2.

that the genotype of bovine $\alpha$ chain mimics that of human $\alpha 2$. First, plasma $\mathrm{Hp} \alpha$ chain displays a molecular weight of 16.7 $\mathrm{kDa}$, similar to that of human $\alpha 2$ on a Western blot (Fig. 5).
Second, the isolated bovine Hp possesses a $\alpha$ chain that is almost identical to that of human in its molecular weight (18.2 kDa) (Fig. 6). Third, by putative amino-acid sequence alignment, 


\begin{tabular}{|c|c|c|c|c|}
\hline$\alpha_{1}$ & A & B & C & \\
\hline $\boldsymbol{\alpha}_{2}$ & A & B1 & B & C \\
\hline
\end{tabular}

$\begin{array}{ccc}A=11 & \text { amino acid residues } \\ (1-11) & B=59 \text { amino acid residues } & C=13 \text { amino acid residues } \\ (12-70) & (71-83)\end{array}$

\begin{abstract}
Human B1 ${ }^{12}$ DDGCPKPPEIAHGYVEHSVRYQCKNYYKLRTEGDGVYTLNDKKQWINKAVGDKLPECEA
Human B ${ }^{71}$ DDGCPKPPEIAHGYVEHSVRYQCKNYYKLRTEGDGVYTLNNEKQWINKAVGDKLPECEA

Identities $=57 / 59(96 \%)$
\end{abstract}

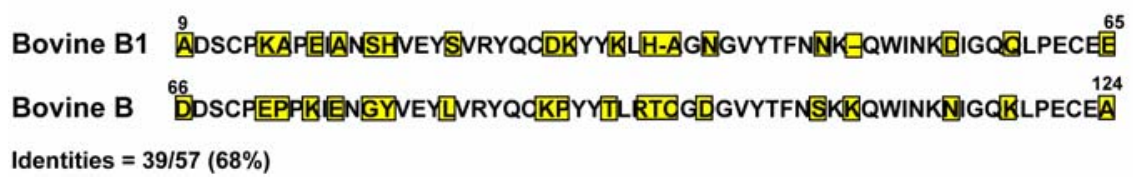

Fig. 10. Schematic drawing of the repeated region (B1 and B) of bovine and human $\alpha$ chain. The uniqueness of human $\alpha 2$ is that it not only matches the $\mathrm{ABC}$ domains of $\alpha 1$, but also with an additional insertion of a redundant sequence (B1 and B repeats). Each repeat unit contains 59 amino-acid residues between Asp-12 to Ala-70. The sequence homology in the repeat region of human is $96 \%$ with two amino acids mutated (in shaded bracket). In cows, there is also a redundant sequence (B1 and B) existing within the $\alpha$ chain, but the sequence homology between the two repeat units (B1 and B) is approximately $68 \%$.

bovine $\alpha$ chain contains a unique redundant sequence, although the sequence homology between the repeated sequences (B1 and B in Fig. 10) was only $68 \%$ as compared to $96 \%$ in human.

It has been previously postulated that the molecular form of bovine $\mathrm{Hp}$ is extremely large, which cannot enter into the gel for SDS-PAGE (Eckersall and Conner, 1990). However, the molecular mechanism involved in the formation of the heterogeneous sizes of bovine $\mathrm{Hp}$ has not been elucidated (Morimatsu et al., 1991). In human, the $\alpha 2$ chain consists of 7 total -SH groups, in which four of them form intra-molecular linkages. Among the other 3 -SH, one (Cys-131) at the $-\mathrm{COOH}$ terminus always internally crosslinks the $\beta$ chain forming a basic $(\alpha 2-\beta)$ subunit, while the other 2 "free" -SH groups are responsible for crosslinking the other two $(\alpha 2-\beta)$ subunits on the $\alpha 2$ chain. As a result, human $\mathrm{Hp} 2-2$ forms cyclic polymers starting with a minimal trimer $(\alpha 2-\beta)_{3}$ or $(\alpha 2-\beta)_{n}$ (where $n \geq 3$ ) as shown in Fig. 1. Remarkably interesting, from the cDNA sequence we show that the $\alpha 2$ chain of bovine is comprised of an additional -SH group (Cys-97) forming a total of $8-\mathrm{SH}$ (Fig. 9). The role of this extra -SH is currently unknown, and we hypothesize that this extra group may form up to 3 "free" -SH in each $\alpha$ chain which is responsible for further polymerization with the other $(\alpha 2-\beta)$ subunits resulting in an even larger molecular form of Hp as compared with human $\mathrm{Hp} \mathrm{2-2} \mathrm{(Fig.} \mathrm{7).} \mathrm{This} \mathrm{notion} \mathrm{is}$ consistent to our hemoglobin typing gel depicted in Fig. 8. Thus, it could explain why the reported values of molecular weight of intact bovine $\mathrm{Hp}$ are extraordinarily large and could not enter the gel when routine SDS-PAGE was conducted (Eckersall and Conner, 1990).
Evolution. It has been well documented that almost all the animals possess $\mathrm{Hp} \mathrm{1-1}$ as the only phenotype (Bowman, 1993). Based on the molecular weight of bovine Hp, some reports have pointed out that bovine $\mathrm{Hp}$ is an exception with a phenotype mimicking human Hp 2-2 (Eckersall and Conner, 1990; Morimatsu et al., 1991; Morimatsu et al., 1992; Yoshino et al., 1992; Katnik et al., 1998). Other ruminant Hp also putatively belongs to this category (Eckersall and Conner, 1990), but the protein has not yet been shown to contain the tandem repeat of $\alpha$ chain on the molecular level. Regardless, it has been thought that humans originally had a single Hp 1-1 phenotype (Smithies et al., 1962). Maeda et al. proposed that the repeated sequence of human $\alpha 2$ had evolved from a nonhomologous unequal crossover between two $\boldsymbol{H p} \mathbf{1}$ genes [Hp 1S and Hp 1F] (Maeda et al., 1984). The uniqueness of Hp 2 allele is that it is present only in humans and not found in all the primates including the new and old world monkeys, chimpanzees, and gorillas (McEvoy and Maeda, 1988). Because the bovine $\alpha$ chain also consists of a $2 / 3 \alpha$ chain repeat similar to humans, our data suggest that the unequal crossover in human is no longer unique. It is of interest to point out that the unique bovine $\alpha 2 \beta$ gene ( $\boldsymbol{H p}$ 2) might exist in cattle as a sole genotype during evolution, since there is no Hp 1-1 or Hp 2-1 genotype identified thus far. We show that approximately $30 \%$ of the repeated region of bovine $\alpha 2$ chain underwent mutation. Thus, the evolved crossover leading to tandem repeat in cow could occur much earlier than that in humans. It seems that this "inserted region" in cows is not well reserved via evolution and its physiologic advantage remains elusive. Alternatively, the bovine Hp 2-2 gene may have pre-existed and not have been evolved from the crossover of Hp 1-1, which is a subject 
of challenge. Nonetheless, the resulting point mutation produced an extra -SH group (Cys-97) that makes bovine $\mathrm{Hp}$ even a larger polymeric form than human $\mathrm{Hp}$ 2-2 (Fig. 7). Hp has been considered as an anti-inflammatory molecule. In humans, the large polymers of $\mathrm{Hp} \mathrm{2-2}$ are a risk in the development of diabetic nephropathy (Nakhoul et al., 2001). One explanation is that the large polymer dramatically retards the penetration into the extracellular space (Nakhoul et al., 2001). We have although recently reported that $\mathrm{Hp}$ is an extremely potent antioxidant, the potency of Hp 2-2 is differentially less than that of 2-1 and 1-1 (Tseng et al., 2004b).

In conclusion, we have shown that although the bovine recombinant $\mathrm{Hp}$ is in a single polypeptide form from the $E$. coli expression system, the antibodies prepared against the $\alpha \beta$ chain can be utilized for the characterization of the molecular forms and isolation of native $\mathrm{Hp}$. The purified bovine $\mathrm{Hp}$ classified, via an immunoaffinity column chromatography and HPLC gel-filtration, is polymeric and heterogeneous in size with molecular weights markedly larger than that of human Hp 2-2. One additional -SH group found in the repeat region of bovine $\alpha$ chain is probably responsible for further cross-linking among the $\alpha-\beta$ subunits in forming complicated Hp polymers. Due to a repeat sequence on bovine $\alpha$ chain that mimics the human $\alpha 2$, we defined bovine Hp genotype to be Hp 2-2, and its presence is not unique in humans.

Acknowledgments This work was supported by the Grant NSC 95-2313-B-009-003-MY2 from the National Science Council, Taiwan, ROC. We thank Drs. Chuen-Huei Shiau and Yi-Ping Lu of the County Livestock Disease Control Center of Pingtung for kindly providing us with bovine plasma.

\section{References}

Bowman, B. H. (1993) Haptoglobin; in Hepatic plasma proteins, Bowman, B. H. (eds.), pp. 159-167, Academic Press, San Diego, USA.

Chan, J. P., Chu, C. C., Fung, H. P., Chuang, S. T., Lin, Y. C., Chu, R. M. and Lee, S. L. (2004) Serum Haptoglobin Concentration in Cattle. J. Vet. Med. Sci. 66, 43-46.

Chen, W. L., Huang, M. T., Liu, H. C., Li, C. W. and Mao, S. J. T. (2004) Distinction between dry and raw milk using monoclonal antibodies prepared against dry milk proteins. J. Dairy. Sci. 87, 2720-2729.

Conner, J. G., Eckersall, P. D., Wiseman, A., Aitchison, T. C. and Douglas, T. A. (1988) Bovine acute phase response following turpentine injection. Res. Vet. Sci. 44, 82-88.

Dobryszycka, W. (1997) Biological functions of haptoglobin--new pieces to an old puzzle. Eur. J. Clin. Chem. Clin. Biochem. 35, 647-654.

Eckersall, P. D. and Conner, J. G. (1990) Plasma haptoglobin in cattle (Bos taurus) exists as polymers in association with albumin. Comp. Biochem. Physiol. B. 96, 309-314.

Emamzadeh, A. R., Hosseinkhani, S., Sadeghizadeh, M., Nikkhah, M., Chaichi, M. J. and Mortazavi, M. (2006) cDNA cloning, expression and homology modeling of a luciferase from the firefly Lampyroidea maculata. J. Biochem. Mol. Biol. 39, 578585.

Gervois, P., Kleemann, R., Pilon, A., Percevault, F., Koenig, W., Staels, B. and Kooistra, T. (2004) Global suppression of IL-6induced acute phase response gene expression after chronic in vivo treatment with the peroxisome proliferator-activated receptor\{alpha\} activator fenofibrate. J. Biol. Chem. 279, 16154-16160.

Hochberg, I., Roguin, A., Nikolsky, E., Chanderashekhar, P. V., Cohen, S. and Levy, A. P. (2002) Haptoglobin phenotype and coronary artery collaterals in diabetic patients. Atherosclerosis 161, 441-446.

Katnik, I., Pupek, M. and Stefaniak, T. (1998) Cross reactivities among some mammalian haptoglobins studied by a monoclonal antibody. Comp. Biochem. Physiol. B Biochem. Mol. Biol. 119, 335-340.

Kristiansen, M., Graversen, J. H., Jacobsen, C., Sonne, O., Hoffman, H., Law, S. K. A. and Moestrup, S. K. (2001) Identification of the haemoglobin scavenger receptor. Nature 409, 198-201.

Labarga, A., Anderson, M., Valentin, F. and Lopez, R. (2005) Web services at European Bioinformatics Institude, EMBnet.news, 11, 18-23.

Laemmli, U. K. (1970) Cleavage of structural proteins during the assembly of the head of bacteriophage T4. Nature 227, 680-685.

Lai, I. H., Tsai, T. I., Lin, H. H., Lai, W. Y. and Mao, S. J. T. (2007) Cloning and expression of human haptoglobin subunits in Escherichia coli: Delineation of a major antioxidant domain. Protein Expr. Purif. 52, 356-362.

Lange, V. (1992) Haptoglobin polymorphism--not only a genetic marker. Anthropol. Anz. 50, 281-302.

Langlois, M. R. and Delanghe, J. R. (1996) Biological and clinical significance of haptoglobin polymorphism in humans. Clin. Chem. 42, 1589-1600.

Levy, A. P., Roguin, A., Hochberg, I., Herer, P., Marsh, S., Nakhoul, F. M. and Skorecki, K. (2000) Haptoglobin phenotype and vascular complications in patients with diabetes. N. Engl. J. Med. 243, 969-970

Liau, C. Y., Chang, T. M., Pan, R. B., Chen, W. H. and Mao, S. J. T. (2003) Purification of human plasma haptoglobin by hemoglobinaffinity column chromatography. J. Chromatogr. B Analyt. Technol. Biomed. Life Sci. 790, 209-216.

Lowry, O. H., Rosebrough, N. J., Farr, A. L. and Randall, R. J. (1951) Protein measurement with Folin phenol reagent. J. Biol. Chem. 193, 265-275.

Maeda, N., Yang, F., Barnett, D. R., Bowman, B. H. and Smithies, O. (1984) Duplication within the haptoglobin Hp2 gene. Nature 309, 131-135.

Maeda, N. (1985) Nucleotide sequence of the haptoglobin and haptoglobin-related gene pair. The haptoglobin-related gene contains a retrovirus-like element. J. Biol. Chem. 260, 6698-6709.

McEvoy, S. M. and Maeda, N. (1988) Complex events in the evolution of the haptoglobin gene cluster in primates. J. Biol. Chem. 263, 15740-15747.

Miyoshi, H., Ohshiba, S., Matsumoto, A., Takada, K., Umegaki, E. and Hirata, I. (1991) Haptoglobin prevents renal dysfunction associated with intravariceal infusion of ethanolamine oleate. $\mathrm{Am}$. J. Gastroenterol. 86, 1638-1641.

Morimatsu, M., Sarikaputi, M., Syuto, B., Saito, M., Yamamoto, S., Saito, M. and Naiki, M. (1992) Bovine haptoglobin: single radial immunodiffusion assay of its polymeric forms and dramatic rise in acute-phase sera. Vet. Immunol. Immunopathol. 33, 365-372. 
Morimatsu, M., Syuto, B., Shimada, N., Fujinaga, T., Yamamoto, S., Saito, M. and Naiki, M. (1991) Isolation and characterization of bovine haptoglobin from acute phase sera. J. Biol. Chem. 226, 11833-11837.

Nakhoul, F. M., Zoabi, R., Kanter, Y., Zoabi, M., Skorecki, K., Hochberg, I., Leibu, R., Miller, B. and Levy, A.P. (2001) Haptoglobin phenotype and diabetic nephropathy. Diabetologia 44, 602-604.

Petersen, H. H., Nielsen, J. P. and Heegaard, P. M. (2004) Application of acute phase protein measurements in veterinary clinical chemistry. Vet. Res. 35, 163-187.

Pillai, S., Silventoinen, V., Kallio, K., Senger, M., Sobhany, S., Tate, J., Velankar, S., Golovin, A., Henrick, K., Rice, P., Stoehr, P. and Lopez, R. (2005) SOAP-based services provided by the European Bioinformatics Institute. Nucleic Acids Res. 33, W25-28.

Raijmakers, M. T., Roed, E. M., te Morsche, R. H., Steegers, E. A. and Peters, W. H. (2003) Haptoglobin and its association with the HELLP syndrome. J. Med. Genet. 40, 214-216.

Schultze, H. E. and Heremans, J. F. (1966) Nature and metabolism of extracellular proteins; in Molecular biology of human proteins, pp. 384-402, Elsevier, Amsterdam,

Smithies, O., Connell, G. E. and Dixon, G. H. (1962) Chromosomal rearrangements and the evolution of haptoglobin genes. Nature 196, 232-236.

Song, C. Y., Chen, W. L., Yang, M. C., Huang, J. P. and Mao, S. J. T. (2005) Epitope mapping of a monoclonal antibody specific to bovine dry milk: involvement of residues $66-76$ of strand D in thermal denatured beta-lactoglobulin. J. Biol. Chem. 280, 35743582.

Tseng, C. F., Huang, H. Y., Yang, Y. T. and Mao, S. J. T. (2004a) Purification of human haptoglobin 1-1, 2-1, and 2-2 using monoclonal antibody affinity chromatography. Protein Expr. Purif. 33, 265-273.

Tseng, C. F., Lin, C. C., Huang, H. Y., Liu, H. C. and Mao, S. J. T. (2004b) Antioxidant role of human haptoglobin. Proteomics 4,
2221-2228.

Wang, S. H., Yang, T. S., Lin, S. M., Tsai, M. S., Wu, S. C. and Mao, S. J. T. (2002) Expression, characterization, and purification of recombinant porcine lactoferrin in Pichia pastoris. Protein Expr. Purif. 25, 41-49.

Wang, Y., Kinzie, E., Berger, F. G., Lim, S. K. and Baumann, H. (2001) Haptoglobin, an inflammation-inducible plasma protein. Redox Rep. 6, 379-385.

Wejman, J. C., Hovsepian, D., Wall, J. S., Hainfeld, J. F. and Greer, J. (1984) Structure and assembly of haptoglobin polymers by electron microscopy. J. Mol. Biol. 174, 343-368.

Wicher, K.B. and Fries, E. (2004) Prohaptoglobin is proteolytically cleaved in the endoplasmic reticulum by the complement C1r-like protein. Proc. Natl. Acad. Sci. USA 101, 14390-14395.

Xu, G., Chen, X., Wu, D., Shi, S., Wang, J., Ding, R., Hong, Q., Feng, Z., Lin, S. and Lu, Y. (2006a) Development of highspecificity antibodies against renal urate transporters using genetic immunization. J. Biochem. Mol. Biol. 39, 696-702.

Xu, L., Wu, W., Zhao, Z., Shao, H., Liu, W., Liu, H. and Li, W. (2006b) Cooperation between human DAF and CD59 in protecting cells from human complement-mediated lysis. $J$. Biochem. Mol. Biol. 39, 743-748.

Yang, S. J. and Mao, S. J. T. (1999) Simple high-performance liquid chromatographic purification procedure for porcine plasma haptoglobin. J. Chromatogr. B. Biomed. Sci. Appl. 731, 395-402.

Yoshino, K., Katoh, N., Takahashi, K. and Yuasa, A. (1992) Purification of a protein from serum of cattle with hepatic lipidosis, and identification of the protein as haptoglobin. Am. J. Vet. Res. 53, 951-956.

Yueh, S. C. H., Lai, Y. A., Chen, W. L., Hsu, H. H. and Mao, S. J. T. (2007) An improved method for haptoglobin 1-1, 2-1, and 2-2 purification using monoclonal antibody affinity chromatography in the presence of sodium dodecyl sulfate. J. Chromatogr. B Analyt. Technol. Biomed. Life Sci. 845, 210-217. 